Supplemental Table 1. Concentrations of anthocyanins of wild blueberry and Concord grape ${ }^{1}$.

\begin{tabular}{|c|c|c|c|}
\hline Peak No. $^{2}$ & Anthocyanin & $\mathrm{mg} / \mathbf{1 0 0 g}\left(\mathrm{FW}^{3}\right)$ & $\%$ of total $\mathrm{ACN}$ \\
\hline \multicolumn{4}{|c|}{ Blueberry (Wild) } \\
\hline 1 & Delphinidin 3-galactoside & 47.6 & $9.8 \%$ \\
\hline 2 & Delphinidin 3-glucoside & 51.2 & $10.5 \%$ \\
\hline 3 & Cyanidin 3-galactoside & 21.9 & $4.5 \%$ \\
\hline 4 & Delphinidin 3-arabinoside & 29.5 & $6.1 \%$ \\
\hline 5 & Cyanidin 3-glucoside & 22.2 & $4.6 \%$ \\
\hline 6 & Petunidin 3-galactoside & 26.2 & $5.4 \%$ \\
\hline 7 & Cyanidin 3-arabinoside & 12.7 & $2.6 \%$ \\
\hline 8 & Petunidin 3-glucoside & 36.1 & $7.4 \%$ \\
\hline 9 & Peonidin 3-galactoside & 7.8 & $1.6 \%$ \\
\hline 10 & Petunidin 3-arabinoside & 14.1 & $2.9 \%$ \\
\hline 11 & Peonidin 3-glucoside & 16.9 & $3.5 \%$ \\
\hline 12 & Malvidin 3-galactoside & 44.0 & $9.0 \%$ \\
\hline 13 & Malvidin 3-glucoside & 64.4 & $13.2 \%$ \\
\hline 14 & Malvidin 3-arabinoside & 24.2 & $5.0 \%$ \\
\hline 16 & Cyanidin 3-(6"-acetyl)galactoside & 1.4 & $0.3 \%$ \\
\hline 18 & Petunidin + pentose & 1.8 & $0.4 \%$ \\
\hline 21 & Delphinidin 3-(6"-acetyl)glucoside & 12.9 & $2.6 \%$ \\
\hline 23 & Cyanidin 3-(6"-acetyl)glucoside & 6.9 & $1.4 \%$ \\
\hline 24 & Malvidin 3-(6"-acetyl)galactoside & 8.1 & $1.7 \%$ \\
\hline 25 & Petunidin 3-(6"-acetyl)glucoside & 9.4 & $1.9 \%$ \\
\hline 26 & Peonidin 3-(6"-acetyl)glucoside & 5.3 & $1.1 \%$ \\
\hline 27 & Malvidin 3-(6"-acetyl)glucoside & 22.0 & $4.5 \%$ \\
\hline \multicolumn{4}{|c|}{ Concord Grape } \\
\hline 1 & Delphinidin + pentose & 0.1 & $0.1 \%$ \\
\hline 2 & Delphinidin 3,5-diglucoside & 3.1 & $2.6 \%$ \\
\hline 4 & Cyanidin 3,5-diglucoside & 1.5 & $1.3 \%$ \\
\hline 5 & Petunidin 3,5-diglucoside & 1.6 & $1.3 \%$ \\
\hline 6 & Delphinidin 3-glucoside & 30.1 & $25.1 \%$ \\
\hline 7 & Peonidin 3,5-diglucoside & 1.0 & $0.8 \%$ \\
\hline 8 & Malvidin 3,5-glucoside & 0.9 & $0.7 \%$ \\
\hline 9 & Cyanidin 3-glucoside & 11.0 & $9.2 \%$ \\
\hline 11 & Petunidin 3-glucoside & 5.8 & $4.8 \%$ \\
\hline 13 & Peonidin 3-glucoside & 1.7 & $1.4 \%$ \\
\hline 14 & Malvidin 3-glucoside & 2.9 & $2.4 \%$ \\
\hline 15 & Cyanidin + pentose & 0.2 & $0.2 \%$ \\
\hline 16 & Delphinidin 3-(6"-acetyl)glucoside & 5.4 & $4.5 \%$ \\
\hline 17 & Delphinidin 3-(6"-coumaroyl)-5-diglucoside & 6.9 & $5.7 \%$ \\
\hline 18 & Cyanidin 3-(6"-acetyl)glucoside & 1.4 & $1.2 \%$ \\
\hline 19 & Cyanidin 3-(6"-coumaroyl)-5-diglucoside & 2.1 & $1.7 \%$ \\
\hline 20 & Delphinidin + coumaroyl + hexose & 0.8 & $0.6 \%$ \\
\hline 22 & Petunidin 3-(6"-acetyl)glucoside & 3.6 & $3.0 \%$ \\
\hline 23 & Malvidin 3-(coumaroyl)-5-diglucoside & 0.9 & $0.8 \%$ \\
\hline 24 & Peonidin 3-(coumaroyl)-5-diglucoside & 0.6 & $0.5 \%$ \\
\hline 25 & Peonidin 3-(6"-acetyl)glucoside & 0.6 & $0.5 \%$ \\
\hline 26 & Malvidin 3-(6"-acetyl)glucoside & 0.2 & $0.1 \%$ \\
\hline 27 & Delphinidin 3-(6"-coumaroyl)glucoside & 24.3 & $20.2 \%$ \\
\hline 28 & Cyanidin 3-(6"-coumaroyl)glucoside & 7.5 & $6.3 \%$ \\
\hline 29 & Petunidin 3-(6"-coumaroyl)glucoside & 3.9 & $3.3 \%$ \\
\hline 30 & Peonidin 3-(6"-coumaroyl)glucoside & 0.8 & $0.7 \%$ \\
\hline 31 & Malvidin 3-(6"-coumaroyl)glucoside & 1.1 & $1.0 \%$ \\
\hline
\end{tabular}

${ }^{1}$ Only anthocyanins with high enough concentrations of being quantified were presented in this table.

${ }^{2}$ Peak numbers correspond to those in Ref. 24.

${ }^{3} \mathrm{FW}$ : fresh weight. 
Supplemental Table 2. Concentrations of anthocyanins from berries and fruits ${ }^{1}$.

\begin{tabular}{|c|c|c|c|}
\hline Peak No. $^{2}$ & Anthocyanin & $\mathrm{mg} / \mathbf{1 0 0 g}\left(\mathrm{FW}^{3}\right)$ & \% of total $\mathrm{ACN}$ \\
\hline \multicolumn{4}{|c|}{ Apple (Red Delicious) } \\
\hline 1 & Cyanidin 3-galactoside & $10.5 \pm 1.5$ & $85.8 \%$ \\
\hline 3 & Cyanidin 3-arabinoside & $0.5 \pm 0.1$ & $4.1 \%$ \\
\hline 4 & Peonidin 3-galactoside & $0.2 \pm 0.1$ & $1.7 \%$ \\
\hline 5 & Cyanidin 7-arabinoside & $0.6 \pm 0.2$ & $4.7 \%$ \\
\hline 6 & Cyanidin 3-xyloside & $0.5 \pm 0.1$ & $4.4 \%$ \\
\hline \multicolumn{4}{|c|}{ Apple (Gala) } \\
\hline 1 & Cyanidin 3-galactoside & $2.1 \pm 0.7$ & $92.7 \%$ \\
\hline 2 & Cyanidin 3-arabinoside & $0.2 \pm 0.1$ & $7.3 \%$ \\
\hline \multicolumn{4}{|l|}{ Apple (Fuji) } \\
\hline 1 & Cyanidin 3-galactoside & $1.2 \pm 0.6$ & $92.5 \%$ \\
\hline 2 & Cyanidin 3-arabinoside & $0.1 \pm 0.1$ & $7.5 \%$ \\
\hline \multicolumn{4}{|l|}{ Blackberry } \\
\hline 1 & Cyanidin 3-glucoside & $198.1 \pm 67.4$ & $76.2 \%$ \\
\hline 2 & Cyanidin 3-arabinoside & $25.1 \pm 28.8$ & $9.6 \%$ \\
\hline 3 & Cyanidin 3-rutinoside & $20.1+20.0$ & $030 \%$ \\
\hline 4 & Pelargonidin 3-glucoside & $0.7 \pm 0.1$ & $0.3 \%$ \\
\hline 5 & Cyanidin 3-(3"-malonyl)glucoside & $0.5 \pm 0.1$ & $0.2 \%$ \\
\hline 7 & Cyanidin 3-xyloside & $13.7 \pm 0.1$ & $5.3 \%$ \\
\hline 8 & Cyanidin 3-(6"-malonyl)glucoside & $5.9 \pm 4.3$ & $2.3 \%$ \\
\hline 9 & Cyanidin 3-dioxalylglucoside & $16.0 \pm 0.3$ & $6.2 \%$ \\
\hline \multicolumn{4}{|c|}{ Marion Blackberry } \\
\hline 1 & Cyanidin 3-glucoside & 217.6 & $72.4 \%$ \\
\hline 2 & Cyanidin 3-rutinoside & 70.8 & $23.6 \%$ \\
\hline 3 & Pelargonidin 3-glucoside & 1.7 & $0.6 \%$ \\
\hline 4 & Peonidin 3-rutinoside & 1.1 & $0.3 \%$ \\
\hline 5 & Cyanidin 3-xyloside & 0.4 & $0.1 \%$ \\
\hline 6 & Cyanidin 3-(6"-malonyl)glucoside & 2.4 & $0.8 \%$ \\
\hline 7 & Cyanidin 3-dioxalylglucoside & 6.5 & $2.2 \%$ \\
\hline \multicolumn{4}{|c|}{ Blueberry (Cultivated) } \\
\hline 1 & Delphinidin 3-galactoside & $61.1 \pm 26.2$ & $15.6 \%$ \\
\hline 2 & Delphinidin 3-glucoside & $19.6 \pm 7.7$ & $5.0 \%$ \\
\hline 3 & Cyanidin 3-galactoside & $14.9 \pm 14.6$ & $3.8 \%$ \\
\hline 4 & Delphinidin 3-arabinoside & $39.4 \pm 5.1$ & $10.1 \%$ \\
\hline 5 & Cyanidin 3-glucoside & $4.1 \pm 2.3$ & $1.1 \%$ \\
\hline 6 & Petunidin 3-galactoside & $34.5 \pm 13.8$ & $8.8 \%$ \\
\hline 7 & Cyanidin 3-arabinoside & $7.4 \pm 4.4$ & $1.9 \%$ \\
\hline 8 & Petunidin 3-glucoside & $15.2 \pm 6.2$ & $3.9 \%$ \\
\hline 9 & Peonidin 3-galactoside & $5.6 \pm 4.6$ & $1.4 \%$ \\
\hline 10 & Petunidin 3-arabinoside & $19.1 \pm 2.4$ & $4.9 \%$ \\
\hline 11 & Peonidin 3-glucoside & $28.6 \pm 7.7$ & $7.3 \%$ \\
\hline 12 & Malvidin 3-galactoside & $41.8 \pm 11.2$ & $10.7 \%$ \\
\hline \multirow[t]{2}{*}{13} & Malvidin 3-glucoside & $34.9 \pm 12.9$ & $8.9 \%$ \\
\hline & Delphinidin ACN & $1.6 \pm 0.2$ & $0.4 \%$ \\
\hline 14 & Malvidin 3-arabinoside & $45.9 \pm 5.9$ & $11.7 \%$ \\
\hline \multirow[t]{2}{*}{18} & Petunidin + pentose & $1.9 \pm 0.5$ & $0.5 \%$ \\
\hline & Penonidin ACN & $0.2 \pm 0.2$ & $0.1 \%$ \\
\hline 23 & Cyanidin 3-(6"-acetyl)glucoside & $2.5 \pm 1.5$ & $0.6 \%$ \\
\hline 24 & Malvidin 3-(6"-acetyl)galactoside & $3.3 \pm 2.2$ & $0.8 \%$ \\
\hline 25 & Petunidin 3-(6"-acetyl)glucoside & $2.3 \pm 1.2$ & $0.6 \%$ \\
\hline 27 & Malvidin 3-(6"-acetyl)glucoside & $6.9 \pm 3.3$ & $1.8 \%$ \\
\hline
\end{tabular}




\section{Supplemental Table 2. (continued)}

Peak No. Anthocyanin

$\mathrm{mg} / \mathbf{1 0 0 g}(\mathrm{FW})$

\% of total ACN

Cherry (Sweet)

1 Cyanidin 3-glucoside

$7.6 \pm 3.0$

$6.2 \%$

2 Cyanidin 3-rutinoside

$105.4 \pm 19.1 \quad 86.4 \%$

4 Pelargonidin 3-rutinoside

6 Peonidin 3-rutinoside

Cranberry

1 Peonidin 3,5-digalactoside

Cyanidin 3-galactoside

Delphinidin 3-arabinoside

Cyanidin 3-glucoside

Pelargonidin 3-galactoside

Cyanidin 3-arabinoside

Peonidin 3-galactoside

Pelargonidin 3-arabinoside

$10 \quad$ Peonidin 3-glucoside

13 Malvidin 3-arabinoside

Grape (Red)

1 Delphinidin 3-glucoside

$1.4 \pm 0.2$

$1.2 \%$

$7.5 \pm 1.9$

$6.1 \%$

$0.3^{4}$

$0.2 \%$

$39.5 \pm 10.2$

$28.0 \%$

$0.2^{4}$

$0.1 \%$

$0.9 \pm 0.2$

$0.7 \%$

$0.7 \pm 0.1$

$0.5 \%$

$25.7 \pm 6.2 \quad 18.2 \%$

$48.6 \pm 9.6 \quad 34.5 \%$

$0.2^{4} \quad 0.1 \%$

$3.5 \pm 1.0$

$2.5 \%$

$20.0 \pm 3.2$

$14.2 \%$

$1.3 \pm 0.8$

$0.9 \%$

$1.1 \pm 0.8$

$3.4 \%$

Cyanidin 3-glucoside

Petunidin 3-glucoside

$3.9 \pm 1.6$

$12.3 \%$

$1.0 \pm 0.9$

$3.3 \%$

Peonidin 3-glucoside

$10.1 \pm 4.5$

$31.9 \%$

Malvidin 3-glucoside

Delphinidin 3-(6"-coumaroyl)glucoside

Malvidin 3-(6"-acetyl)glucoside

$4.8 \pm 3.4$

$15.2 \%$

$0.1^{4}$

0.4

$0.6^{4}$

2.0

$0.3^{4}$

1.0

Petunidin 3-(6"-coumaroyl)glucoside

$0.4^{4}$

1.1

$9.3 \pm 3.3$

$29.3 \%$

11 Malvidin 3-(6"-coumaroyl)glucoside

Nectarine
1 Cyanidin 3-glucoside
2 Cyanidin 3-rutinoside
1 Cyanidin 3-glucoside
1 Cyanidin 3-galactoside
2 Cyanidin 3-glucoside
3 Cyanidin 3-rutinoside
5 Cyanidin 3-(6"-acetyl)glucoside

$6.5 \pm 1.4$

$96.5 \%$

$0.2 \pm 0.1$

$3.5 \%$

Peach

$4.8 \pm 1.2$

$100 \%$

Plum

Plum (Black)

$\begin{array}{cc}1.9 \pm 1.4 & 10.0 \% \\ 12.5 \pm 3.0 & 65.8 \% \\ 4.4 \pm 1.1 & 23.1 \% \\ 0.2 \pm 0.1 & 1.1 \%\end{array}$

1 Cyanidin 3-galactoside

2 Cyanidin 3-glucoside

3 Cyanidin 3-rutinoside

5 Cyanidin 3-(maroyl)glucoside

$7 \quad$ Cyanidin 3-xyloside

8 Cyanidin 3-(6"-acetyl)glucoside

Raspberry (Black)

$\begin{array}{llcc}1 & \text { Cyanidin 3-sambubioside } & 20.8 & 3.0 \% \\ 2 & \text { Cyanidin 3-glucoside } & 87.9 & 12.8 \% \\ 3 & \text { Cyanidin 3-sambubioside-5-rhamnoside } & 157.9 & 23.0 \% \\ 4 & \text { Cyanidin 3-rutinoside } & 402.4 & 58.6 \% \\ 5 & \text { Pelargonidin 3-glucoside } & 1.5 & 0.2 \% \\ 6 & \text { Pelargonidin 3-rutinoside } & 15.2 & 2.2 \% \\ 7 & \text { Peonidin 3-rutinoside } & 1.1 & 0.2 \%\end{array}$

$\begin{array}{cc}0.2 & 0.2 \% \\ 95.6 & 76.8 \% \\ 27.8 & 22.3 \% \\ 0.2 & 0.1 \% \\ 0.4 & 0.4 \% \\ 0.3 & 0.2 \% \\ & \\ 20.8 & 3.0 \% \\ 87.9 & 12.8 \% \\ 157.9 & 23.0 \% \\ 402.4 & 58.6 \% \\ 1.5 & 0.2 \% \\ 15.2 & 2.2 \% \\ 1.1 & 0.2 \%\end{array}$




\section{Supplemental Table 2. (contiuned)}

Peak No. Anthocyanin

$\mathrm{mg} / 100 \mathrm{~g}(\mathrm{FW}) \quad$ \% of total ACN

Raspberry (Red)

1 Cyanidin 3-sophoroside

2 Cyanidin 3-sophoroside-5-rhamnoside

$\begin{array}{cc}32.9 \pm 9.6 & 35.8 \% \\ 24.2 \pm 15.8 & 26.3 \% \\ 19.1 \pm 8.7 & 20.7 \% \\ 14.0 \pm 5.7 & 15.2 \% \\ 1.0 \pm 0.6 & 1.1 \% \\ 0.9 \pm 0.4 & 0.9 \%\end{array}$

\section{Strawberry}

Cyanidin 3-glucoside

Cyanidin 3-rutinoside

Pelargonidin 3-glucoside

Pelargonidin 3-rutinoside

$0.9 \pm 0.4$

$5.6 \%$

Cyanidin 3-glucoside

Cyanidin 3-rutinoside

Pelargonidin 3-glucoside

Pelargonidin 3-rutinoside

$1.2 \pm 0.4$

$0.1^{4}$

$0.3 \%$

$18.2 \pm 3.0$

$84.7 \%$

$1.6 \pm 0.4$

$7.2 \%$

$0.4 \pm 0.1$

$1.6 \%$

5 Pelargonidin 3-(malonyl)glucoside

6 Pelargonidin 3-(6"-acetyl)glucoside

$0.1 \pm 0.1$

$0.6 \%$

\section{Strawberry-OSC}

\begin{tabular}{llcc}
1 & Cyanidin 3-sophoroside & 5.6 & $13.3 \%$ \\
2 & Cyanidin 3-glucoside & 3.1 & $7.5 \%$ \\
3 & Cyanidin 3-rutinoside & 0.7 & $1.7 \%$ \\
4 & Pelargonidin 3-glucoside & 26.3 & $62.9 \%$ \\
5 & Petunidin 3-glucoside & 1.0 & $2.3 \%$ \\
6 & Pelargonidin 3-rutinoside & 1.2 & $2.8 \%$ \\
7 & Pelargonidin 3-(malonyl)glucoside & 3.3 & $7.9 \%$ \\
8 & Pelargonidin 3-(6"-acetyl)glucoside & 0.7 & $1.6 \%$ \\
\hline
\end{tabular}

\footnotetext{
${ }^{1}$ Only anthocyanins with high enough concentrations of being quantified were presented in this table. Data expressed $\mathrm{mg}$ of anthocyanin (anthocyanidin glycosides or acylglycosides) and presented as mean \pm SD for sample number $>2$.

${ }^{2}$ Peak numbers correspond to those in Ref. 24.

${ }^{3} \mathrm{FW}$ : fresh weight.

${ }^{4}$ Only one or two in multiple samples contained this anthocyanin.
} 
Supplemental Table 3. Concentration of anthocyanins in vegetables and nuts ${ }^{1}$.

\begin{tabular}{|c|c|c|c|}
\hline Peak No. $^{2}$ & Anthocyanin & $\mathrm{mg} / \mathbf{1 0 0 g}\left(\mathrm{FW}^{3}\right)$ & $\%$ of total ACN \\
\hline \multicolumn{4}{|c|}{ Black Bean $^{4}$} \\
\hline 1 & Delphinidin 3,5-diglucoside & 0.5 & $1.1 \%$ \\
\hline 2 & Petunidin 3,5-diglucoside & 3.8 & $8.6 \%$ \\
\hline 3 & Delphinidin 3-galacoside & 1.1 & $2.4 \%$ \\
\hline 4 & Delphinidin 3-glucoside & 16.9 & $38.1 \%$ \\
\hline 5 & Malvidin 3,5-diglucoside & 4.1 & $9.2 \%$ \\
\hline 6 & Petunidin 3-galactoside & 0.3 & $0.7 \%$ \\
\hline 7 & Petunidin 3-glucoside & 11.3 & $25.4 \%$ \\
\hline 8 & Malvidin 3-galactoside & 0.2 & $0.5 \%$ \\
\hline 9 & Malvidin 3-glucosie & 6.3 & $14.2 \%$ \\
\hline \multicolumn{4}{|l|}{ Eggplant } \\
\hline 1 & Delphinidin 3-rutinoside-5-galacoside & 11.8 & $13.8 \%$ \\
\hline 2 & Delphinidin 3-rutinoside-5-glucoside & 1.8 & $2.0 \%$ \\
\hline 3 & Delphinidin 3-glucoside & 0.6 & $0.7 \%$ \\
\hline 4 & Delphinidin 3-rutinoside & 71.5 & $83.5 \%$ \\
\hline \multicolumn{4}{|c|}{ Red Leaf Lettuce } \\
\hline 1 & Cyanidin 3-glucoside & $0.09 \pm 0.04$ & $3.9 \%$ \\
\hline 2 & Cyanidin 3-(3"-malonyl)glucoside & $0.08 \pm 0.01$ & $3.5 \%$ \\
\hline 3 & Cyanidin 3-(6"-malonyl)glucoside & $2.03 \pm 1.35$ & $91.1 \%$ \\
\hline 4 & Cyanidin 3-(6"-acetyl)glucoside & $0.03 \pm 0.01$ & $1.5 \%$ \\
\hline \multicolumn{4}{|l|}{ Red Onion } \\
\hline 1 & Cyanidin 3-(6"-malonyl)-glucoside-5-glucoside & 0.3 & $0.5 \%$ \\
\hline 2 & Cyanidin 3-glucoside & 6.7 & $13.8 \%$ \\
\hline 3 & Cyanidin 3-laminaribioside & 3.4 & $7.1 \%$ \\
\hline 4 & Cyanidin 3-(3"-malonylglucoside) & 1.0 & $2.1 \%$ \\
\hline 5 & Peonidin 3-glucoside & 0.8 & $1.7 \%$ \\
\hline 6 & Cyanidin 3-(3"-acetyl)glucoside & 0.1 & $0.3 \%$ \\
\hline 7 & Cyanidin 3-(6"-malonylglucoside) & 23.2 & $47.8 \%$ \\
\hline 8 & Cyanidin 3-(6"-malonyl-laminaribioside) & 11.2 & $23.1 \%$ \\
\hline 9 & Peonidin 3-(malonyl)glucoside & 1.3 & $2.6 \%$ \\
\hline 10 & Cyanidin 3-(malonyl)(acetyl)glucoside & 0.4 & $0.9 \%$ \\
\hline \multicolumn{4}{|c|}{ Small Red Bean } \\
\hline 1 & Cyanidin 3-glucoside & 1.9 & $27.8 \%$ \\
\hline 2 & Pelargonidin 3-sambubioside & 4.1 & $61.9 \%$ \\
\hline 3 & Pelargonidin 3-glucoside & 0.7 & $10.1 \%$ \\
\hline \multicolumn{4}{|l|}{ Pistachio $^{4}$} \\
\hline 1 & Cyanidin 3-galactoside & $6.3 \pm 1.2$ & $84.2 \%$ \\
\hline 2 & Cyanidin 3-glucoside & $1.2 \pm 0.4$ & $15.8 \%$ \\
\hline
\end{tabular}

${ }^{1}$ Only anthocyanins with concentration above the limits of detection were quantified and presented in this table. Data expressed mg of anthocyanin (anthocyanidin glycosides or acylglycosides) and presented as mean \pm SD for sample number $>2 .{ }^{2}$ Peak numbers correspond to those in Ref. 25.

${ }^{3} \mathrm{FW}$ : fresh weight.

${ }^{4}$ Data expressed as dry weight. 


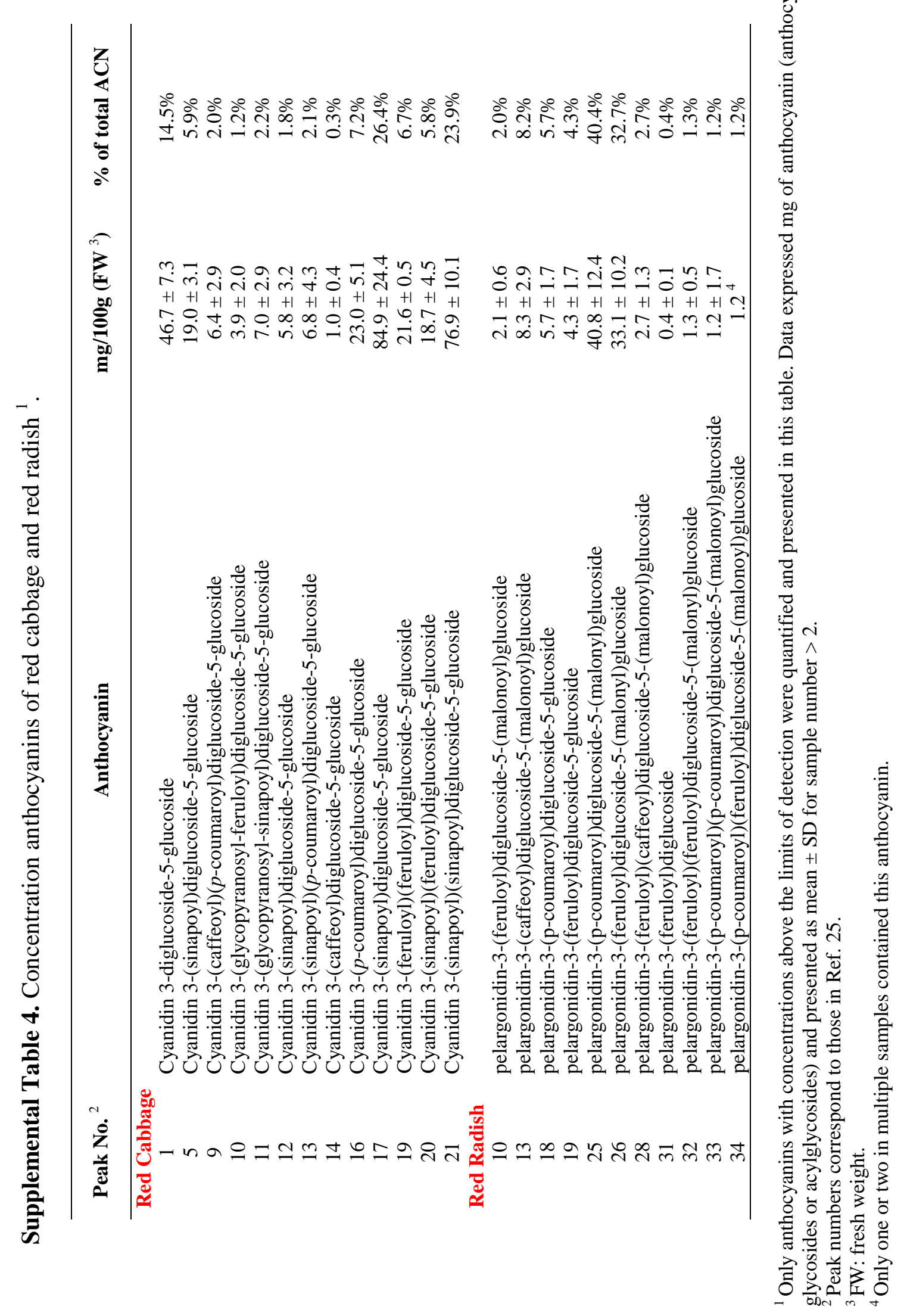

